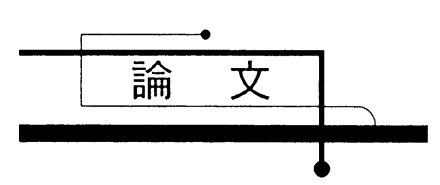

\title{
Study of Ultrafine Bubble Stabilization by Organic Material Adhesion*
}

\author{
Kou SUGANO $^{* *} \quad$ Yuichi MIYOSHI ${ }^{* *} \quad$ Sachiko INAZATO ${ }^{* * *}$
}

\begin{abstract}
We confirmed the effect of organic material adhesion as ultrafine bubble (UFB) stabilization mechanism. Organic material was added to generate UFB, and the state of UFB particles was investigated by dynamic light scattering method, transmission electron microscope (TEM), resonance mass measurement method. Disappearance of UFB was suppressed by the addition of organic material, and it was confirmed that the organic material adhered to the surface of UFB. We got the conclusion that adhesion of organic material inhibit gas dissolution from bubbles.
\end{abstract}

Keywords: Ultrafine bubble, Organic material, Liquid in situ TEM observation

\section{Introduction}

Fine bubbles can be generated by cavitation using venturi tube or swirling flow, and water becomes opaque due to generation of many micro- and milli-bubbles. When the opaque liquid is left over with the microbubble generator off, the bubble rises to the surface level due to the buoyancy or contracts and disappears in the water and the liquid returns to transparent water. When this transparent water is measured by dynamic light scattering method, the existence of ultrafine bubble (UFB) having a diameter of about 100 to $200 \mathrm{~nm}$ may be confirmed. It is reported that this UFB exists stably for over 1 month [1]. However, the mechanism by which UFB stabilizes in the water remains to be unknown, and several theories have been proposed [1-10], but a clear mechanism has not yet been established in reality.

In the dynamic light scattering method, it is difficult to clarify the mechanism that bubbles and solid particles cannot be distinguished from each other. In order to promote the elucidation of the mechanism, a device configuration with high cleanliness is required as much as possible. Various liquid impurities may be involved in the occurrence of UFB. The authors developed a UFB generator in clean environment excluding solid particles and metal ions and tried to elucidate the stabilization mechanism of UFB in order to promote UFB's industrial use. As a result, we got the findings on the UFB stabilization mechanism which suppresses the annihilation of the bubble and report it here.

\section{Model of stabilization mechanism}

The authors confirmed the phenomenon that UFB concentration gradually decreased without reproducing at the stage of constructing the clean generator. Details are described in Section 4. It was also found that the generated UFB was stable without change in concentration even after several days. Since the UFB concentration gradually decreases, some substance is eluted from the wetted member, which acts on the bubble and the UFB concentration also decreases as the elution amount decreases, the model

* 2017.5.19 受付

** Engineering Division Automotive \& Industrial Systems Company Panasonic Corporation, Japan 1006 Kadoma, Kadoma City, Osaka 571-8506 TEL: +81-50-3587-3718 E-mail: sugano.k@jp.panasonic.com

*** Innovation Promotion Sector Advanced Research Division Panasonic Corporation, Japan 
of the UFB stabilization mechanism shown in Fig. 1 is inferred.

Normally, bubbles generated by cavitation contract and disappear (Fig. 1, upper row), but if any eluted material is present, it adheres to the bubble. Although the bubble shrinks, adhered material covers the entire bubble and stabilizes with a particle size of several hundred $\mathrm{nm}$ or less in diameter (Fig. 1, lower row). The bubble takes the route of shrinkage, that is, the gas within the bubble elutes into the surrounding liquid. Adhered substances are thought to inhibit this route. This model has not only the dynamic equilibrium model [1] but also the feature of the skin model [2] covering the whole bubble.

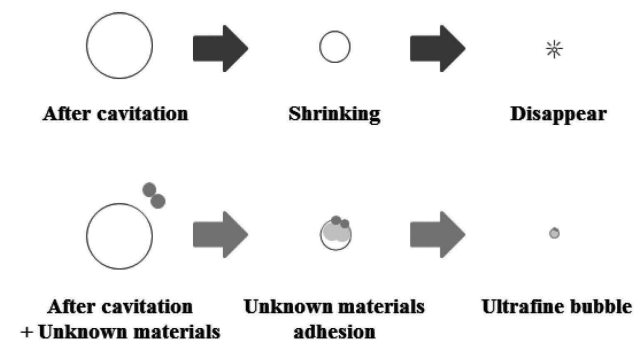

Fig. 1 UFB stabilization model.

\section{Experimental method}

The configuration of the UFB generator used in the experiment for verifying the UFB stabilization model is shown in Fig. 2. The generator was installed in a class 10 clean booth and UPW (ultra-pure water, specific resistance value $18 \mathrm{M} \Omega \cdot \mathrm{cm}$ ) was used as the water to be supplied. Dissolved oxygen concentration of UPW is $4 \sim 5 \mathrm{mg} / \mathrm{L}$ (Experimental environment measurement value), and UFB water produced in one experiment is $60 \mathrm{~mL}$. UPW sealed in the generator was circulated by a pump and passed through a cavitation nozzle to generate UFB. In order to prevent contamination, the UFB generator adopts a method of generating UFB from dissolved gas without providing an open water tank or a mechanism for sucking gas. Therefore, it is not UFB generation in air supersaturated state. The impeller of the pump was magnetically levitated and had no dust generation, and the part to be wetted was made of fluorocarbon resin material except for EPDM made O-ring. The pressure value was measured by installing a pressure gauge at the point immediately before the cavitation nozzle [P1] and immediately before the pump [P2]. The UFB concentration was measured after releasing the pressure inside the generator using a NanoSight LM-10 manufactured by Malvern Co., Ltd., which is a method of collecting UFB water from the Sample Port and analyzing the Nano Tracking Analysis method. The pressure inside the generator was released beforehand so that the influence on gas dissolution due to pressure change was made as small as possible. Measurement was made 15 minutes after the pump driving time at which concentration change stabilized. The temperature of the liquid was measured by installing a thermocouple just before the cavitation nozzle; the initial values of $25 \sim 27^{\circ} \mathrm{C}$ changed only to $37 \sim 40^{\circ} \mathrm{C}$ after 15 minutes $(\Delta t=12$ $\sim 13^{\circ} \mathrm{C}$ ).

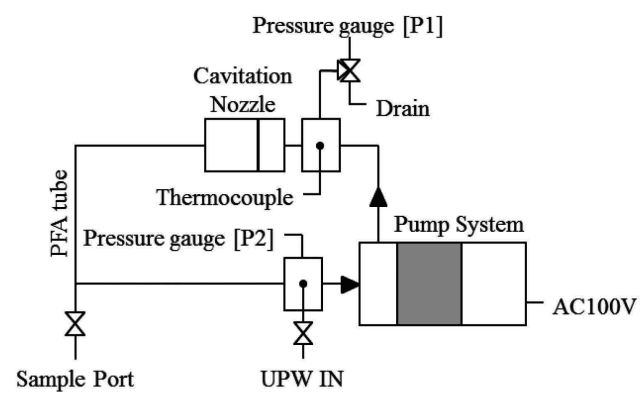

Fig. 2 Construction of UFB generator.

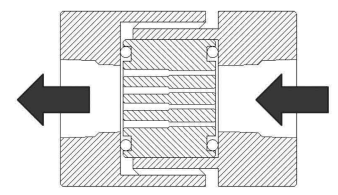

Fig. 3 Cavitation nozzle schematic draw.

The cavitation nozzle shown in Fig. 3 is connected to a $3 / 8$-inch PFA pipe with a joint, so it is inserted in the case and sealed with an O-ring. The nozzle body is a cylinder, a diameter of $25.6 \mathrm{~mm}$ and a height of $20 \mathrm{~mm}$. Water is pressurized by the pump, passes through the PFA piping, is narrowed by the cavitation nozzle, the flow velocity increases, the pressure decreases, the pressure returns to the PFA pipe diameter on the inlet side, cavitation occurs and bubbles are generated. The definition formula of the Reynolds number $R e$ is shown below. 


$$
R e=\frac{V L}{v}
$$

Here, $V$ is the relative average speed of the flow, $L$ is the characteristic length, and $v$ is the kinematic viscosity coefficient. $R e$ in this experimental system was $[23,600]$. The definition formula of the cavitation number $\sigma$ is shown below.

$$
\sigma=\frac{2(P-P v)}{d V^{2}}
$$

Here, $P$ is the absolute pressure, $P v$ is the vapor pressure, $d$ is the density of the fluid, $V$ is the representative velocity of the flow, and $\sigma$ in this experimental system is [0.4]. It is more effective for cavitation generation to increase $L$ in order to increase the Reynolds number, but general machines the height of the nozzle body was set at $20 \mathrm{~mm}$ from the limit of processing technology. Table 1 shows operating conditions such as pressure, temperature, and flow rate at the time of UFB generation. In this paper, we assume that UFB concentration is measured by NanoSight LM - 10 .

Table 1 UFB generation operating condition.

\begin{tabular}{ccl}
\hline \hline Generation time & 15 & $\mathrm{~min}$ \\
$\begin{array}{c}\text { Pump rotation speed } \\
\text { Liquid pressure [P1] }\end{array}$ & 8000 & $\mathrm{rpm}$ \\
Initial pressure & 0 & $\mathrm{kPa}[\mathrm{G}]$ \\
Exit pressure & $180 \sim 200$ & $\mathrm{kPa}[\mathrm{G}]$ \\
Liquid pressure [P2] & & \\
Initial pressure & 0 & $\mathrm{kPa}[\mathrm{abs}]$ \\
After 5 minutes & $-15 \sim-20$ & $\mathrm{kPa}[\mathrm{abs}]$ \\
Exit pressure & $30 \sim 37$ & $\mathrm{kPa}[\mathrm{abs}]$ \\
Liquid temperature & & \\
Initial temperature & $25 \sim 27$ & degree C \\
Exit temperature & $37 \sim 40$ & degree C \\
Liquid flow & $7.1 \sim 8.0$ & $\mathrm{~L} / \mathrm{min}$ \\
\hline \hline
\end{tabular}

\section{Results}

\subsection{UFB generation number dependency}

Details of the UFB concentration lowering phenomenon mentioned in the section 2 above is described below.

A decrease in UFB concentration was confirmed with the repetition of UFB production in the apparatus. The UFB generation procedure is shown below, but it was found that the procedure (1) to (5) was carried out as a set and it was carried out 8 times, the UFB concentration gradually generated decreased as shown in Fig. 4. The nozzle through hole was measured after the experiment, but the dimensions did not change. This is not the effect of nozzle structure change due to erosion.

Experimental procedure for UFB generation

(1) UPW introduced

(2) Pump ON (Circulation for 15 minutes)

(3) Pump OFF

(4) UFB water sampling and measurement

(5) Channel cleaning

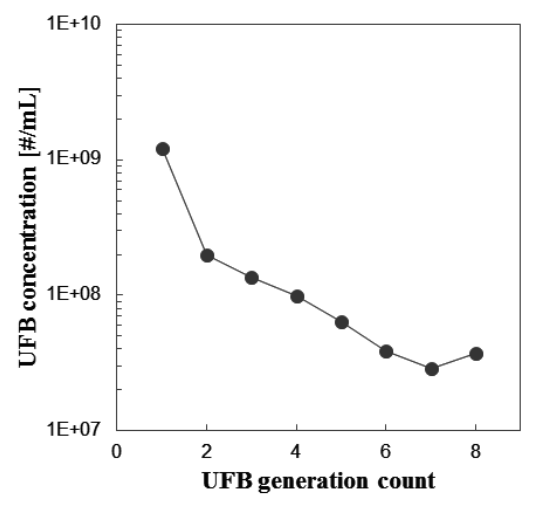

Fig. 4 Transition of UFB concentration on generation count.

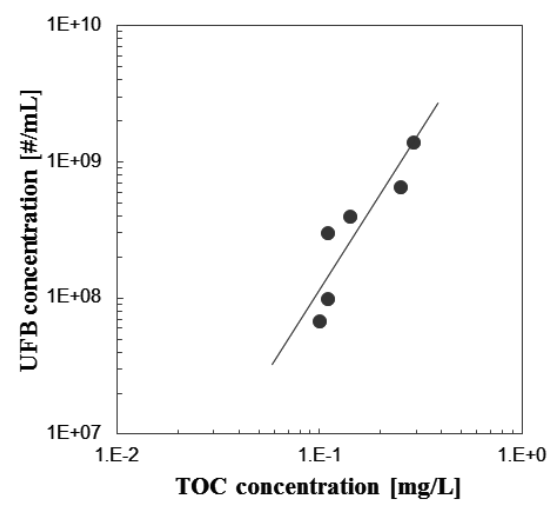

Fig. 5 Relationship between TOC concentration and UFB concentration.

Elution from fluorocarbon resin materials is well known in processes requiring care of foreign materials and metals like semiconductors. It is common to use acid cleaning beforehand to let the eluate dry before use [11]. In this experiment, UFB was generated without cleaning the fluorocarbon resin 
with acid, so it is considered that some eluted material is present from the wetted member and affects UFB generation. In the above experiments, the amount of elution decreased, and as a result it was considered that the UFB concentration decreased. In order to confirm this, the eluate of UFB water obtained in the experiment was analyzed. The UFB water shown in Fig. 4 was analyzed for inorganic and organic material by Inductively Coupled Plasma-Mass Spectrometry (ICP-MS), Ion Chromatography (IC) and Total Organic Carbon (TOC). As a result, it was found that the TOC concentration correlated with the UFB concentration. Fig. 5 shows the results of TOC analysis of UFB water with the number of experiments 1 to 6 in Fig. 4. Analysis of this organic substance itself was not carried out, but it showed that the organic material in the liquid acts on UFB and contributes to UFB concentration.

\subsection{Effect of addition of organic material on UFB concentration}

In order to verify the influence of TOC on UFB concentration, UFB was generated by adding organic material to UPW in advance. The organic material was quantitatively added from the Drain port upstream of the cavitation nozzle. Table 2 shows the list of organic materials used in the experiment. Fig. 6 shows the result of adding these organic materials and generating UFB. There was no dependence of TOC concentration on IPA and HMDS. We think this is due to the small molecular weight and short carbon chain. However, UFB concentration tended to increase with Heptane and Dodecane depending on the added concentration, and data indicating that TOC in liquid contributes to suppression of UFB annihilation was obtained. It was also found that UFB concentration decreased as the addition exceeded a certain amount, and peaked with respect to added amount. Hydrocarbon has a surfactant effect and becomes self-micellar when it exceeds the critical micelle concentration, so the action on UFB decreases and the UFB concentration decreases. Note that the self-micellarized hydrocarbons are considered to have a size of $50 \mathrm{~nm}$ or less in diameter, so they cannot be detected by the Nano Tracking Analysis method.
Table 2 List of added organic materials.

Organic material Chemical formula

\begin{tabular}{ll}
\hline \hline IPA & $\mathrm{C}_{3} \mathrm{H}_{8} \mathrm{O}$ \\
HMDS & $\mathrm{C}_{6} \mathrm{H}_{18} \mathrm{OSi}_{2}$ \\
Heptane & $\mathrm{C}_{7} \mathrm{H}_{16}$ \\
Dodecane & $\mathrm{C}_{12} \mathrm{H}_{26}$ \\
Oleic Acid & $\mathrm{C}_{18} \mathrm{H}_{34} \mathrm{O}_{2}$ \\
a-tocopherol & $\mathrm{C}_{29} \mathrm{H}_{50} \mathrm{O}_{2}$ \\
\hline \hline
\end{tabular}

From the results shown in Fig. 6, the UFB concentration tended to be higher in organic material with larger number of carbons and long carbon chain. Next, oleic acid which is a long chain fatty acid was added to generate UFB. The results are shown in Fig. 7. The addition of $100 \mathrm{ppm}$ resulted in a high concentration of $1.1 \times 10^{10} / \mathrm{mL}$, indicating that the effect of suppressing UFB extinction was remarkable. The generated particle diameter did not differ even when the addition amount became 10 times, and the peak particle diameter was around $80 \mathrm{~nm}$ in diameter.

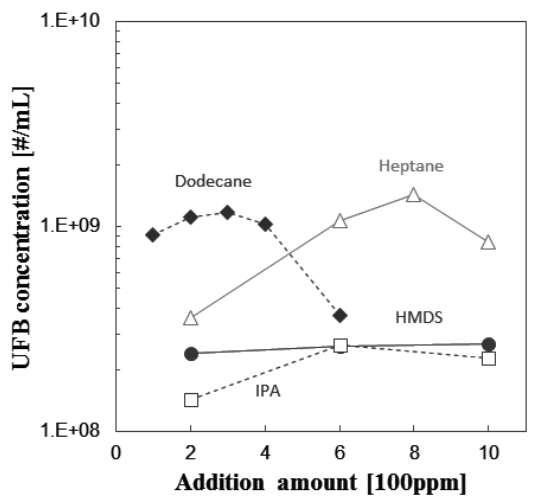

Fig. 6 Dependence of UFB concentration on addition amount of various organic materials.

In the particle size distribution diagram shown in Fig. 8, the particle distribution range did not change and no phenomenon of particle aggregation was observed. This graph is obtained by enlarging the 0 to $300 \mathrm{~nm}$ portion from the original graph upper right Fig. 9 shows the change in concentration when this UFB water is left standing at room temperature in a clean room, and Fig. 10 shows the particle size 


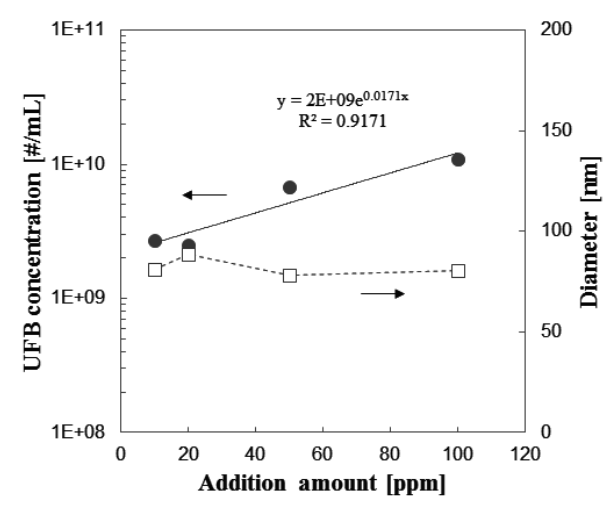

Fig. 7 Dependence of UFB concentration and diameter on addition amount of oleic acid.

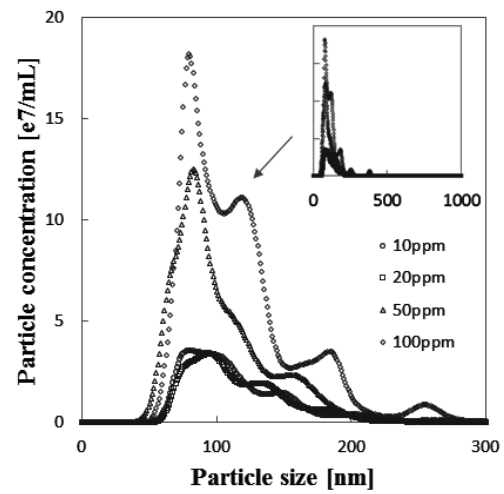

Fig. 8 Particle size distribution to the addition amount of oleic acid.

distribution. Evaluation was made through 76th day, but there are some variations in concentration, but there is no remarkable change. In the particle size distribution, it was confirmed that it is in the same range as after 0 day and it is a stabilized UFB. It was confirmed that it was stabilized UFB even in the particle size distribution range. In addition, almost the same result was obtained when long-chain vitamin $\mathrm{E}$ ( $\alpha$-tocopherol) was added.

It is considered from this experiment that bubbly extinction can be suppressed by adding organic material.

\subsection{UFB analysis by TEM and resonant mass measurement}

A new technique was developed to directly observe UFB with a transmission electron microscope (TEM)

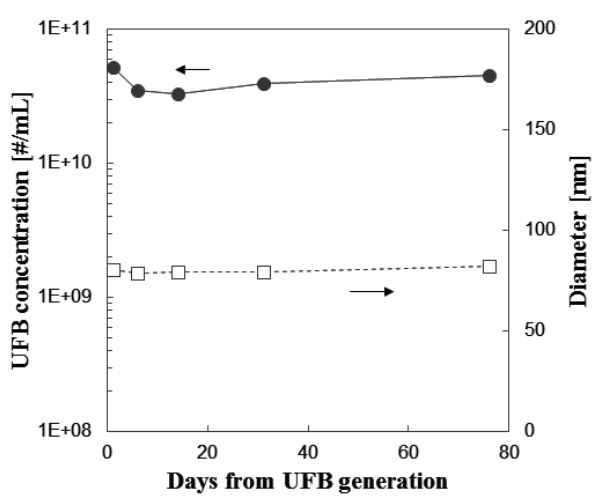

Fig. 9 Time dependence of oleic acid added UFB concentration and diameter.

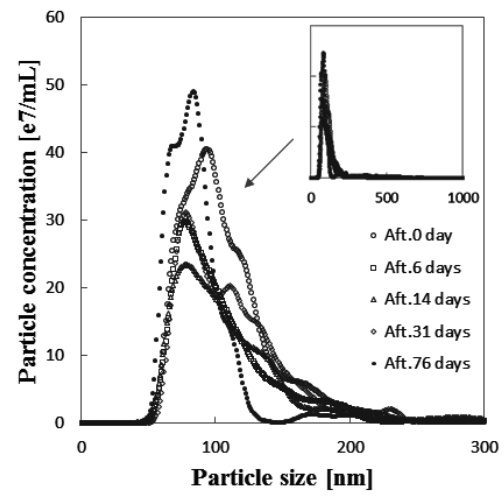

Fig. 10 Particle size distribution over time of oleic acid added UFB.

[12]. TEM observation of organic material added UFB produced using this technique. A MEMS (Micro Electro Mechanical Systems) chip is mounted on an in-situ holder of TEM. UFB water is introduced into the MEMS chip to make a very thin liquid phase of several hundred $\mathrm{nm}$. By this, we observe the particles in the liquid with high resolution. This method is characterized in that UFB can be observed directly as it is without special treatment such as freezing.

TEM image of UFB generated by adding oleic acid to Photo 1 is shown. Photo 2 shows an enlarged image of UFB generated, (a) shows oleic acid, (b) shows vitamin E, and (c) shows adding hydrocarbon to oleic acid. In both cases, adhesion of black material was confirmed on the surface of transparent round particles. This transparent particle is a bubble, and the black material is considered to be an organic material. 


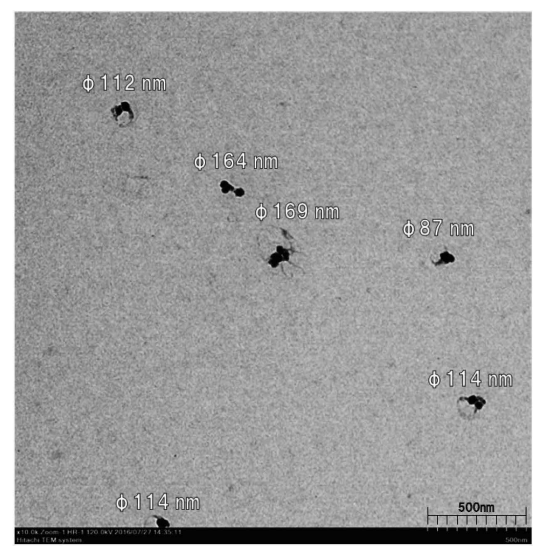

Photo 1 TEM image of oleic acid added UFB.

It was possible to obtain a photograph suggesting that bubbles were stably formed by organic material adhering to the surface of bubbles. The bubbles in Photo 1 is 87 to $169 \mathrm{~nm}$ in diameter. Since the liquid phase depth is $500 \mathrm{~nm}$, the bubble concentration becomes $1.8 \times 10^{12} / \mathrm{mL}$ when calculated from this image. The UFB concentration when this sample is measured by the Nano Tracking Analysis method is $5.2 \times 10^{10} / \mathrm{mL}$, and it is necessary to clarify the difference of the number concentration by the measurement method in the future.

In the TEM image, it looks like air bubbles but it is measured using a resonance mass measurement method (Archimedes manufactured by Malvern) to obtain confirmation. The resonant mass measurement method is characterized in that the resonance frequency varies with the particles passing through the MEMS sensor and laser reflected light from the cantilever chip is mass detected by a photodiode detector, whereby bubbles and solid particles can be distinguished and measured. Specifically, since the cantilever with heavy particles vibrates slowly, they are recognized as solid particles, and the cantilever with light particles vibrates faster, so they are recognized as bubbles. Measurements were made on both microsensor with flow path cross section of $8 \times 8$ $\mu \mathrm{m}$ and $2 \times 2 \mu \mathrm{m}$ nanosensor. In the case of the micro sensor, since the measurement range is $0.5 \mu \mathrm{m}$ or more, the measurement result with a high resolution nanosensor is shown in Fig. 11. As a result, a high positive concentration was detected within the range

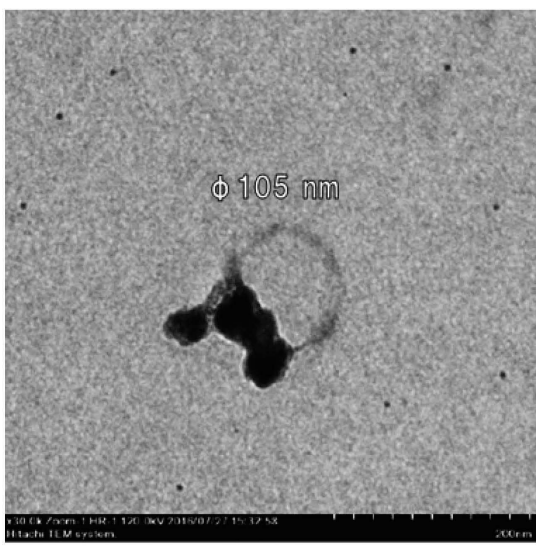

(a) Oleic acid added UFB.

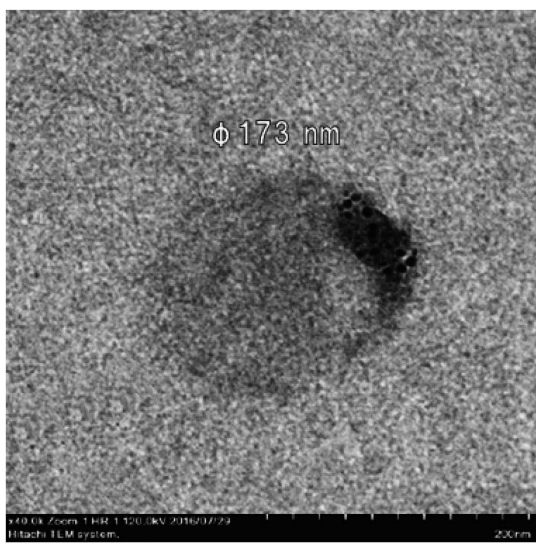

(b) $\alpha$-tocopherol added UFB.

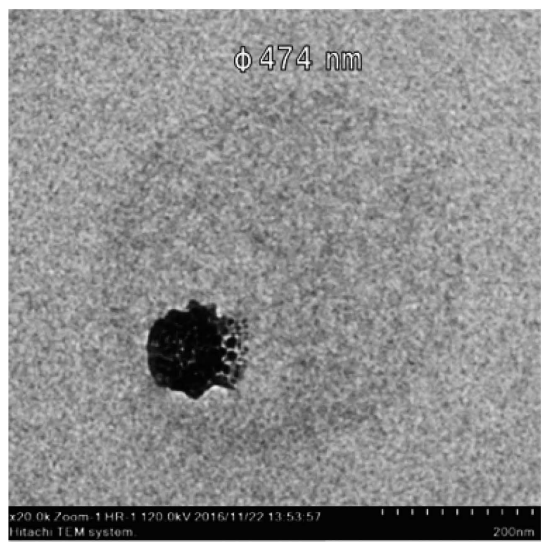

(c) Oleic acid and hydrocarbon added UFB.

Photo 2 TEM image of hydrophobic material added UFB. 
of 0.1 to $0.5 \mu \mathrm{m}$. In addition, the value of UFB number concentration measured by the resonant mass measurement method is $1 / 100-1 / 1000$ of the Nano Tracking Analysis method.

The detected particles are almost counted as bubbles, but since hydrophobic organic materials such as oleic acid are lighter than water, there is a possibility of counting particles of oleic acid alone. However, in the TEM image, particles adhering to the bubbles could be confirmed, and a material which can be regarded as oleic acid simple material particles could not be confirmed. From these facts, it was confirmed that at least the particles confirmed in the TEM image are bubbles. Vitamin E-added UFB water was also measured, but the same result was obtained.

Measurement data provider : Fine Bubble Technology Office National Institute of Technology and Evaluation (NITE).

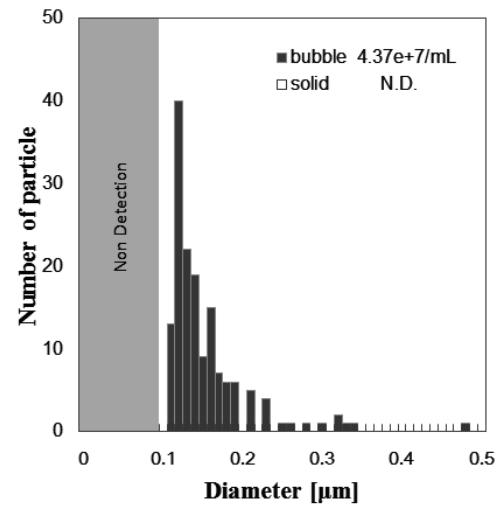

Fig. 11 Particle size distribution of oleic acid added UFB measured by Archimedes.

\section{Consideration on UFB stabilization mechanism}

As described above, in this experiment,

- UFB generation concentration depends on TOC concentration in liquid

- UFB is stabilized even if it is not supersaturated

- Suppression of extinction of UFB by addition of organic material

Results were obtained. From these facts, it was found that organic material act on UFB to suppress disappearance and the UFB is stabilized even if the condition is not such that air bubbles tend to be generated due to the supersaturation state of the air and the gas is hard to dissolve.

One of the mechanisms is a mechanism in which bubbles are stabilized by suppressing the outflux of gas from the bubbles into the water by the organic material adhering to the bubble surface.

TEM analysis and resonant mass spectrometry were carried out to confirm how organic acts on bubbles material. As a result, it was able to confirm that organic material adheres to the bubble surface. Therefore, it became clear that organic material adhering to the bubble surface contributes to stabilization of bubbles.

A dynamic equilibrium model has been proposed as a bubble stabilization mechanism [1]. A schematic diagram of the dynamic equilibrium model predicted in this model is shown in Fig. 12(a). It is found that it is very similar to the bubble shape actually measured by TEM observation. Ordinarily, gas escapes from bubbles into water, but in the dynamic equilibrium model, organic material adheres to a part of bubbles and acts to collect and flow gas dissolved in water to bubbles. Since it causes the action of flowing into the bubbles, it is the theory that air bubbles can exist stably by balancing the influxing amount of gas from this organic material with the amount of gas outfluxing into water from bubbles. Also, as shown in the model in Chapter 2 of this paper, it is considered that the organic material also possesses the feature of the skin model Fig. 12(b) acting as suppression of gas outflux by covering the bubble. Therefore, it is considered that gas dissolution is further inhibited more than the dynamic equilibrium model. In the calculation results, when the bubble is $150 \mathrm{~nm}$ or less in diameter and the covering ratio of organic material is $50 \%$ or more, bubbles can exist in a stabilized state. In this experimental result, the bubble diameter is 87 to $474 \mathrm{~nm}$ and the coverage rate of organic material calculated by comparing areas of organic material and bubbles from photographs by simple grid method is 10 to $25 \%$, so it is different from the bubble shape actually measured this time. This result adhered organic material suggests that also combines features of skin model covering the bubble. However, including the definition of organic particle adhesion 

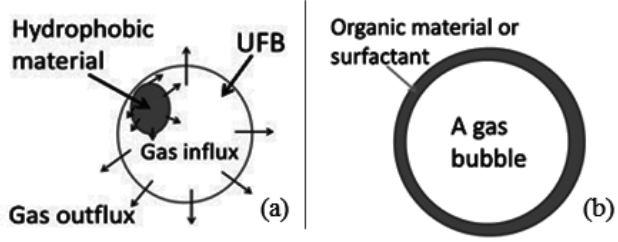

Fig. 12 UFB image of (a) dynamic equilibrium model [1] and (b) skin model [2].

particle size and organic material coverage ratio, it is necessary to examine the consistency of calculation of dynamic equilibrium theory and measurement result in the future. However, we think it significant that bubbles that support the bubble model diagram of the dynamic equilibrium theory could actually be observed. Based on the results of this experiment, we look forward to the future progress of the dynamic equilibrium theory for the foam stabilization mechanism.

\section{Conclusions}

We clarified that TOC is affecting the suppression of UFB extinction and found that disappearance of UFB can be suppressed by adding a small amount of organic material. Furthermore, it was confirmed by TEM observation that organic material adhered to the surface of UFB, and organic material contributed to suppression of UFB extinction. From these facts, the possibility of supporting the dynamic equilibrium theory as a bubble stabilization mechanism was shown.

\section{Acknowledgements}

In preparing this paper, we thank Mitsuru Tanaka of the Fine Bubble Industry Association, Mr. Seika Ouchi of the Fine Bubble Technology Office National Institute of Technology and Evaluation and Mr. Kyuichi Yasui at the National Institute of Advanced Industrial Science and Technology.

\section{Nomenclature}

Re : Reynolds Number

$V$ : Relative average flow velocity

$[\mathrm{m} / \mathrm{s}]$

$L \quad:$ Characteristic length

$P \quad$ : Absolute pressure
$P v \quad:$ Fluid vapor pressure

$d \quad$ : Fluid density

$\left[\mathrm{kg} / \mathrm{m}^{3}\right]$

\section{Greek letters}

$v \quad:$ Kinematic viscosity

$\sigma \quad$ : Cavitation Number

\section{References}

[1] Yasui, K., Mechanism for Stability of Ultrafine Bubbles, Japanese Journal of Multiphase Flow, Vol.30 (1), 19-26 (2016).

[2] Yount, D. E., Skins of Varying Permeability: A Stabilization Mechanism for Gas Cavitation Nuclei, J. Acoust. Soc. Am., Vol. 65, 1429-1439 (1979).

[3] Azmin, M., Mohamedi, G., Edirisinghe, M. and Stride, E. P., Dissolution of Coated Microbubbles: The Effect of Nanoparticles and Surfactant Concentration, Mter. Sci. Eng. C, Vol.32, 2654-2658 (2012).

[4] Strasberg, M., Onset of Ultrasonic Cavitation in Tap Water, J. Acoust. Soc. Am., Vol. 31, 163-176 (1959).

[5] Takahashi, M., $\zeta$ Potential of Microbubbles in Aqueous Solutions: Electrical Properties of the Gas-Water Interface, J. Phys. Chem. B, Vol. 109, 21858-21864 (2005).

[6] Bunkin, N. F. and Bunkin, F. V., Bubston Structure of Water and Aqueous Solutions of Electrolytes, Phys. Wave Phenom., Vol. 21, 81-109 (2013).

[7] Weijs, J. H., Seddon, J. R. T. and Lohse, D., Diffusive Shielding Stabilizes Bulk Nanobubble Clusters, Chem. Phys. Chem, Vol. 13, 2197-2204 (2012).

[8] Okamoto, R. and Onuki, A., Bubble Formation in Water with Addition of a Hydrophobic Solute, Eur. Phys. J. E, Vol. 38, 72 (2015).

[9] Yarom, M. and Marmur, A., Stabilization of Boiling Nuclei by Insoluble Gas: Can a Nanobubble Cloud Exist?, Langmuir, Vol. 31, 7792-7798 (2015).

[10] Yasui, K., Tuziuti, T., Kanematsu, W. and Kato, K., Advanced Dynamic-Equilibrium Model for a Nanobubble and a Micropancake on a Hydrophobic or Hydrophilic Surface, Phys. Rev. E, Vol.91, 033008 (2015).

[11] Takata, T., Determination of Trace Impurities in Highly Purified Reagent, TOSOH Research and Technology Review, Vol. 54 (2010).

[12] Inazato, S., Nakazawa, E., Sakaguchi, Y., Iseki, M., Wayama, M., Nakano, K., Ominai, Y. and Konomi, M., Observation in the Liquid of the Ultrafine Bubble by TEM, The Japanese Society of Microscopy, $72^{\text {nd }}$ Annual Meeting 167 (2016). 